\title{
Descending necrotizing mediastinitis in a child with chickenpox
}

\author{
César Pérez-Caballero Macarrón, MD, ${ }^{a}$ Alfonso Pérez Palomino, MD, ${ }^{a}$ José Antonio Matute de Cárdenas, MD, ${ }^{b}$ \\ Nieves Sanz Villa, MD, ${ }^{\text {b }}$ and Luis Moreno Fernández, MD, ${ }^{a}$ Madrid, Spain
}

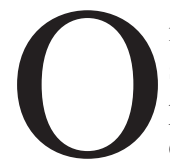

ne of the most dreaded forms of mediastinitis is descending necrotizing mediastinitis (DNM), with a reported mortality of $40 \%$ to $50 \% .^{1}$ It occurs as a complication of infections that arise from odontogenic $(50 \%-60 \%)$ or cervicofascial infections or cervical trauma and can complicate various clinicopathologic conditions. Although the diagnostic characteristics and need for antibiotics are accepted, the type of drainage is controversial. Surgical management, and particularly the optimal form of mediastinal drainage, remains controversial, with support ranging from cervical drainage alone to cervical drainage and routine thoracotomy. We present a clinical case of DNM in a child with chickenpox.

\section{Clinical Summary}

A 4-year-old-boy was admitted to our hospital with severe respiratory distress, cyanosis, fever, tachycardia, hypotension, cutaneous rash on the chest and abdomen, and several residual skin blisters. Five days earlier he had received a diagnosis of chickenpox. Bilateral alveolar infiltrates, enlarged mediastinum, and bilateral pleural effusion were assessed by chest radiography. Empirical treatment with cefotaxime and vancomycin was used for 14 days. Streptococcus pyogenes was found in blood culture. Chest computed axial tomography (CT) showed DNM (Figure 1).

Debridement of necrotic mediastinal tissues from the anterior and middle mediastinum was achieved through a median sternotomy combined with a transverse cervical approach. The thymus was completely excised, along with inflammatory and necrotic tissue surrounding the supra-aortic vessels, larynx, trachea, and the lateral portions of the esophagus down to the infracarinal region. The pericardium was partially excised and left open. Mediastinal drains were left in place in the upper and lower anterior mediatinum as well as in both pleural cavities for continuous drainage of saline washings for 7 days. The sternum was left open and the skin closed. Ten days after the operation, the sternum was closed uneventfully. A residual mediastinal parasternal abscess and two small pleural empyemas were assessed in a new CT scan. Imipenem and amikacine were used for 15 days with a successful radiologic, clinical, and analytic outcome.

From the Pediatric Critical Care Unit ${ }^{\mathrm{a}}$ and the Department of Pediatric Surgery, ${ }^{\mathrm{b}}$ San Rafael Children's Hospital, Madrid, Spain.

Received for publication July 20, 2006; accepted for publication Sept 5, 2006.

Address for reprints: Alfonso Pérez Palomino, MD, Pediatric Critical Care Unit, San Rafael Children's Hospital, Madrid, Spain (E-mail: gonsigonsi@ yahoo.es).

J Thorac Cardiovasc Surg 2007;133:271-2

$0022-5223 / \$ 32.00$

Copyright () 2007 by The American Association for Thoracic Surgery

doi:10.1016/j.jtcvs.2006.09.030
The patient was discharged from the hospital on postoperative day 38. After a follow-up of 6 months, he is doing well and thriving.

\section{Discussion}

DNM is an uncommon form of mediastinitis that can rapidly progress to sepsis. Our case matched the criteria for DNM as defined by Estrera and associates. ${ }^{2}$

DNM has been described as a polymicrobial infection, with participation of aerobic and anaerobic bacteria. Gram positive cocci were the most frequently isolated aerobic bacteria, in accordance with the literature mentioning Streptococcus as the aerobic microorganism most commonly related to mediastinitis. One feature of such infections is their unlimited extensive character, affecting several anatomic zones without respect for anatomic barriers, provoking necrosis of muscles and fascias and inducing systemic toxicity.

Characteristically, the clinical course is dramatic with severe systemic toxicity and alarmingly rapid spread. Mediastinal abscesses associated with DNM are common. Other severe complications such as erosion of the aorta, aortic aneurysm, costal osteomyelitis, and rupture of the innominate artery are described. ${ }^{3}$

Delay of diagnosis is one of the primary reasons for the high mortality in DNM. The diagnosis is suspected when the patient has chest pain, dyspnea, and fever with marked associated toxemia. CT scan can confirm the diagnosis. The treatment of DNM consists of

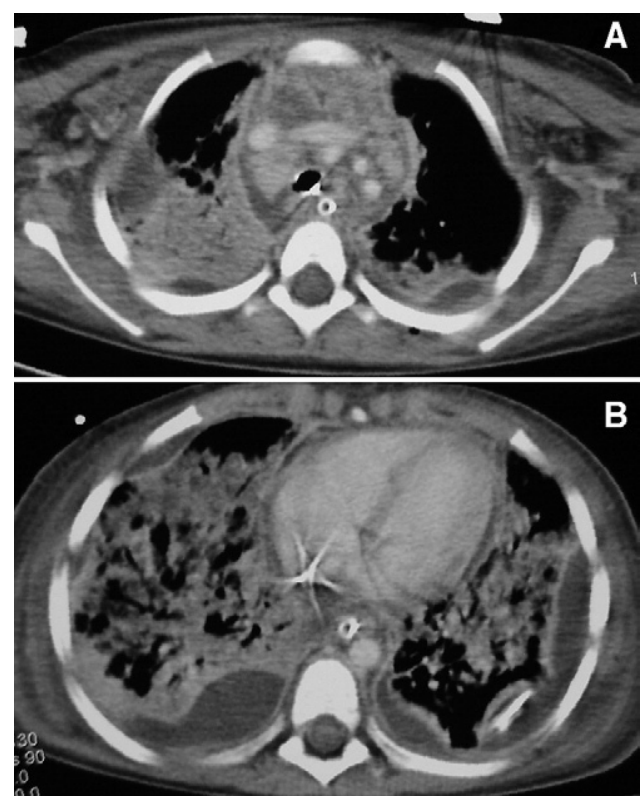

Figure 1. Chest CT scans: A, Predominant anterior and middle mediastinitis. B, Severe pneumonia and pleural effusion. 
intravenous antibiotics, surgical debridement and drainage, and airway management.

Corsten and associates' ${ }^{4}$ meta-analysis demonstrated a significant survival advantage in those patients who were treated by a combined drainage of the neck and mediastinum versus cervical drainage alone. The authors recommend a formal thoracotomy to drain the chest. Brunelli and colleagues, ${ }^{5}$ however, recommend a cervicomediastinal drainage (cervical incision to enter the thorax) of the mediastinum for cases of superior mediastinitis, with a formal thoracotomy reserved for those cases in which the DNM involvement extends beyond the carina.

Our surgical approach through a median sternotomy associated with a transverse cervical incision was based on the fact that the patient had a compromised cardiac output and CT findings consistent with a predominant anterior and middle mediastinitis. The posterior and infracarinal mediastinum were less affected by the disease. Our purpose was to decompress the heart along with a proper debridement. Inotropic requirements after surgery were significantly lower.

Abscesses tend to reform in the days after surgery, and a daily cervical wash is necessary to release collections and to collapse forming abscesses. Irrigation and drainage of the mediastinum are conducted daily.

Management of DNM should always be surgical, with broad, extensive, open cervicotomy together with ample mediastinal drainage and associated thoracotomy, if necessary, to ensure the adequate drainage of all mediastinal compartments.

\section{References}

1. Lavini C, Natali P, Morandi U, Dallari S, Bergamini G. Descending necrotizing mediastinitis: diagnosis and surgical treatment. $J$ Cardiovasc Surg (Torino). 2003;44:655-660.

2. Estrera AS, Landay MJ, Grisham JM, Sinn DP, Platt MR. Descending necrotizing mediastinitis. Surg Gynecol Obstet. 1983;157:545-52.

3. Kruyt PM, Boonstra A, Fockens P, Reeders JW, van Lanschot JJ. Descending necrotizing mediastinitis causing pleuroesophageal fistula. Chest. 1996;109:1404-7.

4. Cortsen MJ, Shamji FM, Odell PF, Frederico JA, Laframboise GG, Reid KR, et al. Optimal treatment of descending necrotising mediastinitis. Thorax. 1997;52:702-8.

5. Brunelli A, Sabbatini A, Catalini G, Fianchini A. Descending necrotizing mediastinitis. Arch Otolaryngol Head Neck Surg. 1996;122:1326-9.

\title{
Technique of right single-lung transplantation for idiopathic pulmonary fibrosis using cross-field ventilation
}

\author{
Seth D. Force, MD, ${ }^{a}$ Andres Pelaez, MD, ${ }^{\mathrm{b}}$ David C. Neujahr, MD, ${ }^{\mathrm{b}}$ Allan M. Ramirez, MD, ${ }^{\mathrm{b}}$ William Whitley, MD, \\ Daniel L. Miller, MD, ${ }^{a}$ and E. Clinton Lawrence, MD, ${ }^{b}$ Atlanta, Ga
}

I solated lung ventilation for single or bilateral sequential lung transplantation can be achieved with either a double-lumen endotracheal tube or a single-lumen endotracheal tube with a bronchial blocker. However, situations might arise that call for alternative methods of ventilation until the airway can be stabilized. We present a patient in whom we were unable to achieve isolated lung ventilation using standard techniques but were successful with cross-field ventilation of the nontransplanted lung during a right single-lung transplantation.

\section{Clinical Summary}

A 54-year-old man presented for lung transplantation evaluation with end-stage lung disease caused by pulmonary fibrosis. A chest

From the Divisions of Cardiothoracic Surgery ${ }^{\mathrm{a}}$ and Pulmonary Medicine ${ }^{\mathrm{b}}$ and the Department of Anesthesiology, ${ }^{\mathrm{c}}$ Emory University, Atlanta, Ga.

Received for publication July 24, 2006; accepted for publication Sept 5, 2006.

Address for reprints: Seth D. Force, MD, The Emory Clinic, Rm 2213, 1365 Clifton Rd, NE Atlanta, GA 30324 (E-mail: seth_force@emoryhealthcare.org).

J Thorac Cardiovasc Surg 2007;133:272-3

$0022-5223 / \$ 32.00$

Copyright $\odot 2007$ by The American Association for Thoracic Surgery doi:10.1016/j.jtcvs.2006.09.013 computed tomographic scan performed as part of the transplantation evaluation showed fibrotic lung disease bilaterally and a significant rightward angulation of the trachea (Figure 1). Bronchoscopy confirmed the severe deviation of the trachea and revealed the right upper lobe bronchus and bronchus intermedius to be coming directly off of the trachea. A quantitative ventilationperfusion scan showed that the right lung was receiving $34 \%$ of the overall blood and air flow, and therefore the patient was listed for right single-lung transplantation.

At the time of transplantation, because of the severe deviation of the patient's trachea, the double-lumen endotracheal tube was unable to be positioned properly. Single-lung ventilation was next attempted by placing a single-lumen endotracheal tube down the left main bronchus, but this was also unsuccessful. The singlelumen tube was then positioned in the midtrachea, and a bronchial blocker was placed into the right mainstem bronchus.

The patient was positioned in the left lateral decubitus position and underwent a standard right posterolateral thoracotomy incision. The recipient pneumonectomy was performed after an extensive lysis of adhesions, and the endotracheal tube was then directed down the left main bronchus. However, it was not possible to place the endotracheal balloon completely within the bronchus, and a large part of the balloon was therefore obstructing the distal trachea. The endotracheal tube was then withdrawn into the midtrachea, and a number 7.0 armored tube was then placed 